\title{
Interdisciplinaridade em Bacharelado no Brasil: O Caso de Relações Internacionais da USP
}

\author{
Daniela Carla Decaro Schettini*, Maria Antonieta Del Tedesco Lins, Marislei Nishijima \\ Instituto de Relações Internacionais da Universidade de São Paulo
}

* Autora para correspondência: danischettini@usp.br

\begin{abstract}
RESUMO
Multidisciplinaridade e interdisciplinaridade são elementos inerentes a muitos cursos novos no Brasil, o que desafia os educadores com formação tradicional em áreas específicas. Este estudo de caso sobre o bacharelado em Relações Internacionais (BRI) da Universidade de São Paulo (USP) pode ser elucidativo sobre os problemas enfrentados para a condução desses novos cursos para uma integração interdisciplinar. O referido curso propõe isonomia de créditos obrigatórios entre suas disciplinas de formação teórica básica e amplia as possibilidades de escolha multidisciplinar com optativas. Este artigo avalia o grau de interdisciplinaridade do curso aplicando mineração de textos aos conteúdos programáticos das suas disciplinas. Os resultados mostram que: a) as disciplinas de formação básica e as optativas são pouco interligadas, embora tenham evoluído no tempo; e b) as disciplinas optativas escolhidas são muito aderentes às das áreas de formação básica. Há de fato um grau modesto de interdisciplinaridade, revelando a dificuldade de consolidar a proposta.
\end{abstract}

Palavras-Chave: Multidisciplinaridade; Interdisciplinaridade; Formação em Relações Internacionais.

\begin{abstract}
Multidisciplinarity and interdisciplinarity are intrinsic elements in numerous undergraduate new courses in Brazil, which challenge educators with traditional training in specific areas. This case study on the bachelor degree in international relations (BRI) of the University of São Paulo (USP) can illustrate the problems faced by faculty and coordinators for an interdisciplinary integration. The BRI degree expects the students to combine compulsory credits of basic theoretical areas with a second part of elegible disciplines to be chosen among a wide range within the basic areas and other possibilities. The article evaluates the degree of interdisciplinarity of the course applying text mining to the disciplines syllabi. The results show that: a) basic and optional training subjects are little interconnected, although they have evolved over time; and b) the chosen elegible disciplines are very adherent to those of the basic theoretical areas. There is in fact a modest degree of interdisciplinarity, revealing the difficulty of consolidating the course's proposal.
\end{abstract}

Keywords: Multidisciplinarity; Interdisciplinarity; Major in International Relations.

\section{Introdução}

Este artigo propõe uma reflexão sobre uma experiência de currículo avaliado como multidisciplinar - entendido aqui como uma forma inicial de composição de um currículo com distintas áreas do conhecimento que se sobrepõem e convivem - do Bacharelado em Relações Internacionais da Universidade de São Paulo (BRI-USP), criado em 2002. Procura-se analisar empiricamente o efetivo grau de proximidade entre as disciplinas que compõem o curso por meio do estudo de seus conteúdos programáticos. Os resultados apontam para um moderado grau de interdisciplinaridade surgido a partir da multidisciplinaridade.

A experiência com a interdisciplinaridade vem despertando interesse crescente neste milênio. Tanto em discursos de dirigentes acadêmicos, como em chamadas para projetos de investigação, o termo interdisciplinaridade passou a aparecer como uma meta a se alcançar. Grande parte das referências à interdisciplinaridade a entendem como uma evolução no processo de produção de conhecimento (LATTUCA, 2001, pp. 7-8; KLEIN, 2005, 2010a, inter alia), embora existam manifestações de ceticismo 
sobre o tema, em detrimento dos benefícios estabelecidos pelas referências disciplinares (e.g.JACOBS, 2014). Numa análise positiva, são perceptíveis o surgimento e a solidificação de número significativo de novas carreiras universitárias e áreas de conhecimento edificadas pela interconexão entre disciplinas "tradicionais", as quais têm como prerrogativa o desafio de compreender e escolher a forma de relacionamento entre as disciplinas que as compõem.

As relações internacionais são, pela própria definição de seu objeto, um diálogo entre disciplinas de diferentes áreas do conhecimento. Ainda que se identifiquem no estudo das guerras as origens das relações internacionais como campo de conhecimento, seria limitante buscar sua fundamentação em apenas uma área científica (SMITH, 2000; RIGUEIRA, 2012, pp. 23-24). Desde os primórdios, esteve em pauta o dilema entre definir uma nova disciplina ou abrigá-la no espectro temático de disciplinas tradicionais (RIGUEIRA, 2012). Colocou-se a questão de estabelecer uma disciplina independente ao mesmo tempo que se formavam laços entre disciplinas tradicionais, cujos objetos de estudo e métodos são relativamente consolidados, criando, de fato, o que passou a ser a área de relações ou estudos internacionais.

A abertura de fronteiras disciplinares gera espaço para criatividade, troca, liberdade na produção científica. Ao mesmo tempo origina uma produção híbrida, decorrente de eventuais miscigenações, resultando em conhecimentos difíceis de serem avaliados e classificados pelas estruturas departamentais existentes, agências de fomento e o mercado de trabalho.

Embora o surgimento de carreiras multi e interdisciplinares conte com mais de uma década no Brasil, há poucas análises sobre essas experiências em relações internacionais (e.g. AALTO, 2011; BLANTON, 2009; RIGUEIRA, 2012; VENTURA \& LINS, 2014). Nesse sentido, estudos de casos podem servir como importantes auferidores da capacidade de interconexão e diálogo entre disciplinas nesses cursos.

Além desta seção, a próxima resume o debate sobre o enquadramento disciplinar das relações internacionais. Em seguida, são apresentados os dados utilizados e a metodologia, seguidos do detalhamento dos resultados e, por fim, da conclusão.

\section{Relações Internacionais Definidas por Relações entre Disciplinas}

No contexto de busca de diálogo entre disciplinas, as interações estabelecidas entre disciplinas com diferentes finalidades não devem ser identificadas diretamente com um padrão uniforme de constituição de novas áreas de conhecimento, no tempo e no espaço. Weingart (2010, p. 4) reflete sobre a história da organização do conhecimento humano, situando o estabelecimento das fronteiras entre as ciências, tal como as conhecemos hoje, no final do século XVIII. Essa classificação tem funções práticas ao organizar em "departamentos" a produção, ao regulamentar ofícios e profissões, ao determinar espaços para pesquisa e ensino. Weingart vai além, afirmando que as disciplinas, em sua organização e relativa estabilidade, desempenham uma função de mediar e dirigir as mudanças sociais na medida em que acumulam e filtram o conhecimento, descartando aquilo que parece não ser mais relevante. A departamentalização disciplinar assume, assim, um papel prático antes de função ou razão epistemológica.

Depois de séculos de primazia da atividade científica disciplinar, principalmente a partir dos anos 1990, surgiram propostas de atividades combinando disciplinas de formas diferentes. As possibilidades de interação ultrapassam as barreiras das grandes áreas como as comumente classificamos: em humanidades, exatas ou ciências da natureza. Uma análise das ciências humanas e sociais de forma ampla dá conta de que:

A organização das áreas de conhecimento não se dá do mesmo modo em todo o mundo, em razão de particularidades históricas e circunstâncias locais, mas sem dúvida há uma valorização da categoria ciência, que designa um tipo de conhecimento que é valorizado em outros domínios e esferas relevantes para o seu futuro desenvolvimento. (VELHO, 2003, p. 16). 
As relações internacionais têm sua história identificada com esse fenômeno, nas diversas partes do mundo em que se estabeleceu como nova "área" ou disciplina. Assim, da mesma forma que o nascimento das relações internacionais enquanto disciplina difere dos padrões das ciências ditas "tradicionais", a classificação da sua produção acadêmica e o desenho dos currículos de formação na área não seguem necessariamente estruturas semelhantes àquelas oferecidas por áreas cujas fronteiras têm delimitações a priori mais precisas (KLEIN, 2005).

Para além da importância relativa das áreas de conhecimento de um currículo de relações internacionais, coloca-se uma questão mais complexa relacionada ao caráter de sua interação: o tipo e a qualidade de convivência entre as áreas na estrutura do curso e o valor das possíveis inter-relações na formação dos alunos. Um caminho seria simplesmente "empilhar" as áreas, materializadas nas disciplinas que as compõem, deixando que as conexões entre elas se façam por meio de temas transversais e do processo de aprendizagem dos estudantes, que entendemos como multidisciplinaridade. Long (2011, p. 38) apresenta uma clara descrição do processo de multidisciplinaridade:

The multidisciplinary challenge emerges because disciplinary subject matters are in fact not clearly separate and distinct; there are overlapping issues or new concerns, patterns of behaviour, configurations of power. Multidisciplinary does not challenge disciplinary paradigms, dominant theories and methods, but simply adds them to one another.

Em meio às possibilidades mais avançadas de integração entre as disciplinas, a interdisciplinaridade poderia ser definida como um diálogo e uma troca de conceitos, conteúdos e métodos entre as disciplinas, conduzindo a experiências novas, a priori inéditas para as disciplinas tradicionais. Uma experiência interdisciplinar seria, por exemplo, estudar temas a partir de abordagens e métodos conjuntos e mesclados com origem em diferentes disciplinas: estabelecendo conteúdos programáticos comuns, introduzindo metodologias compartilhadas ou afins. Aqui se cria algo novo com base na fusão de elementos diversos das diferentes disciplinas, o que se configuraria como uma abordagem transdisciplinar (VENTURA \& LINS, 2014, pp. 108-109).

Ao estabelecer uma taxonomia da interdisciplinaridade, Klein (2010b, p. 16) propõe uma representação gráfica para o alcance dos diferentes níveis de relações entre disciplinas. A multidisciplinaridade seria definida pela justaposição, coordenação e sequenciamento de conceitos e práticas das diversas disciplinas. $\mathrm{O}$ real processo de integração entre conteúdos, teorias e práticas, mesclagem, interação e interconexão só pode ser efetivado pelo exercício da interdisciplinaridade. A transgressão ou transformação é o resultado da transdisciplinaridade.

Para as relações internacionais, o conceito de (inter)disciplinaridade tem grande relevância para a definição de fronteiras na produção de conhecimento e organização acadêmica, o que implica necessariamente o estabelecimento de critérios de classificação e de aferição de mérito à produção (LONG, 2011). Tanto a sua produção científica como a colocação dos egressos no mercado de trabalho enfrentam a dificuldade de enquadramento de uma área que vai além das fronteiras disciplinares.

Do ponto de vista da colaboração entre acadêmicos para a produção conjunta de conhecimento, o exercício da interdisciplinaridade pressupõe um esforço mais intenso dos que a praticam em relação ao conforto da produção monodisciplinar, pois se coloca a tarefa de "dialogar no idioma do outro" no que tange à epistemologia, aos conceitos e métodos. Aalto (2011, p. 69) sintetiza o processo de colaboração entre áreas na interdisciplinaridade:

Our methodological means must lend themselves to research problems of different magnitudes, and vary from more rigorous to looser formats of organization. Organization refers to the patterning of knowledge claims and findings into a more comparable format in which the novelty and import of observations, findings and arguments is better discerned and possible synergies identified. 
Da mesma maneira, a elaboração de programas de disciplinas de um curso de graduação com conteúdo efetivamente interdisciplinar demanda uma preparação muito particular, bastante distinta da concepção de um programa de disciplina tradicional. Além da decisão conjunta do grupo de docentes acerca do conteúdo programático, a sua criação envolve a definição de metodologias de ensino por professores com formação disciplinar clássica num processo complexo e extremamente enriquecedor.

No bacharelado em relações internacionais da USP, apesar do valor atribuído desde o início do curso à pluralidade disciplinar, poucas foram as iniciativas de programas concebidos de forma interdisciplinar (VENTURA \& LINS 2014). O fato de o discurso da interdisciplinaridade e a real atenção ao tema estarem presentes no Instituto inspirou o exercício empírico que aqui apresentamos.

\section{Dados e Métodos}

Para avaliar o grau de interdisciplinaridade do currículo do BRI-USP, usamos técnicas de mineração de textos com cálculo de frequência de palavras e associações de palavras entre textos referentes aos programas de suas disciplinas. Coletamos os conteúdos programáticos das disciplinas, disponíveis no sistema Júpiter da USP, para serem usados como insumos das análises.

Ao finalizarem as disciplinas obrigatórias ao longo dos dois primeiros anos da graduação, os alunos do BRI-USP devem complementar sua formação escolhendo disciplinas optativas eletivas dentro de um espectro bem mais amplo de disciplinas. Estas podem ser oferecidas por docentes do próprio Instituto ou por departamentos das áreas que compõem os pilares formativos dos primeiros anos, a saber: Economia, Política, Direito e História. Finalizadas as disciplinas eletivas, os alunos precisam ainda de créditos em disciplinas optativas livres, que podem ser cursadas em outras áreas ou em programas de intercâmbio.

No segundo segmento do curso de relações internacionais, a escolha é praticamente exclusiva do aluno. Assim, a investigação empírica de suas escolhas por disciplinas, dentro das opções temáticas, importa para a avaliação da interação entre os campos disciplinares no curso. Permite, deste modo, avaliar o grau de multidisciplinaridade mantido pelo aluno em sua formação após a formação de base teórica multidisciplinar obrigatória.

Considerando essas características peculiares do currículo do BRI-USP, propomos dois procedimentos para mensurar o diálogo entre as disciplinas, que entendemos como multidisciplinaridade, mas que tem evoluído para algum grau de interdisciplinaridade. Primeiro, analisamos o conteúdo programático das disciplinas obrigatórias na primeira fase do curso. Em seguida, retraçamos as trilhas seguidas pelos estudantes no segundo momento do curso, quando escolhem as disciplinas optativas.

A análise das disciplinas obrigatórias foi feita em conjunto para identificar relações em seus conteúdos programáticos. Para o caso das disciplinas optativas, isolamo-nas por áreas de estudo e por aluno representativo por ano (desde 2006, com a primeira turma de formados no curso de BRI-USP). Em mais detalhes, para a análise das disciplinas optativas eletivas, construímos a figura do aluno representativo. Definido anualmente, esse aluno é o resultado da média do número de todas as disciplinas cursadas por cada um de nossos alunos regulares. Se, por exemplo, cinco alunos cursaram a disciplina A e três a disciplina $\mathrm{B}$, o aluno representativo cursou oito disciplinas nesse período. Dessa forma, é dado mais peso para as disciplinas que são mais frequentadas.

Para evitar perturbações no exercício, foram retiradas algumas palavras consideradas pouco relevantes $^{1}$, bem como as siglas das disciplinas. Visando a agregar vocábulos com o mesmo significado, outras palavras foram transformadas (Anexo I).

Usamos a ferramenta de análise de texto Voyant ${ }^{2}$, que permitiu elaborar os seguintes conjuntos de mapas de frequência de palavras dos conteúdos programáticos: i) das disciplinas obrigatórias em 2016; ii) das disciplinas optativas por área em 2016; iii) das disciplinas optativas por ano, de 2006 a 2016; e também iv) alguns mapas e gráficos das disciplinas optativas combinados por períodos de anos (inicial, intermediário e final), a fim de identificar tendências na escolha dos alunos. 


\section{Resultados}

\section{Disciplinas Obrigatórias}

A Figura 1 mostra a frequência relativa das cinco palavras mais comuns ("internacional", "estado", "política", "direito" e "economia") nas cinco principais áreas das disciplinas obrigatórias (eixo da abscissa). Elas remetem justamente às cinco principais áreas de conhecimento em que o curso está estruturado, a saber: Direito, Economia, Política, História e Métodos Quantitativos. São exemplos as palavras "estado" e "direito" na área de Direito e "política" e "economia" em suas próprias áreas. Os conteúdos programáticos das áreas de História e Métodos Estatísticos não tiveram palavras específicas de suas áreas com frequência suficiente para aparecer entre as cinco palavras mais frequentes, o que revela que são disciplinas com menor peso na estrutura curricular.
Observa-se também que as disciplinas das áreas de Economia (Direito) consideram questões de política com certa frequência, mas quase não tratam de assuntos relacionados com o Direito (Economia). A área de História quase não considera questões de economia, política e direito. Por outro lado, a área de Política é a mais diversa, pois mantém alta relação com assuntos das disciplinas de Direito e Economia. Por fim, "internacional" é bastante frequente no conteúdo programático de todas as áreas, de acordo com os propósitos do curso.

A Figura 2 ilustra as quinhentas palavras mais frequentes no conteúdo programático das disciplinas obrigatórias. O tamanho da palavra está diretamente relacionado com a sua frequência. "Estado" e "internacional" são as palavras mais frequentes, seguidas de "política", "economia" e "direito".

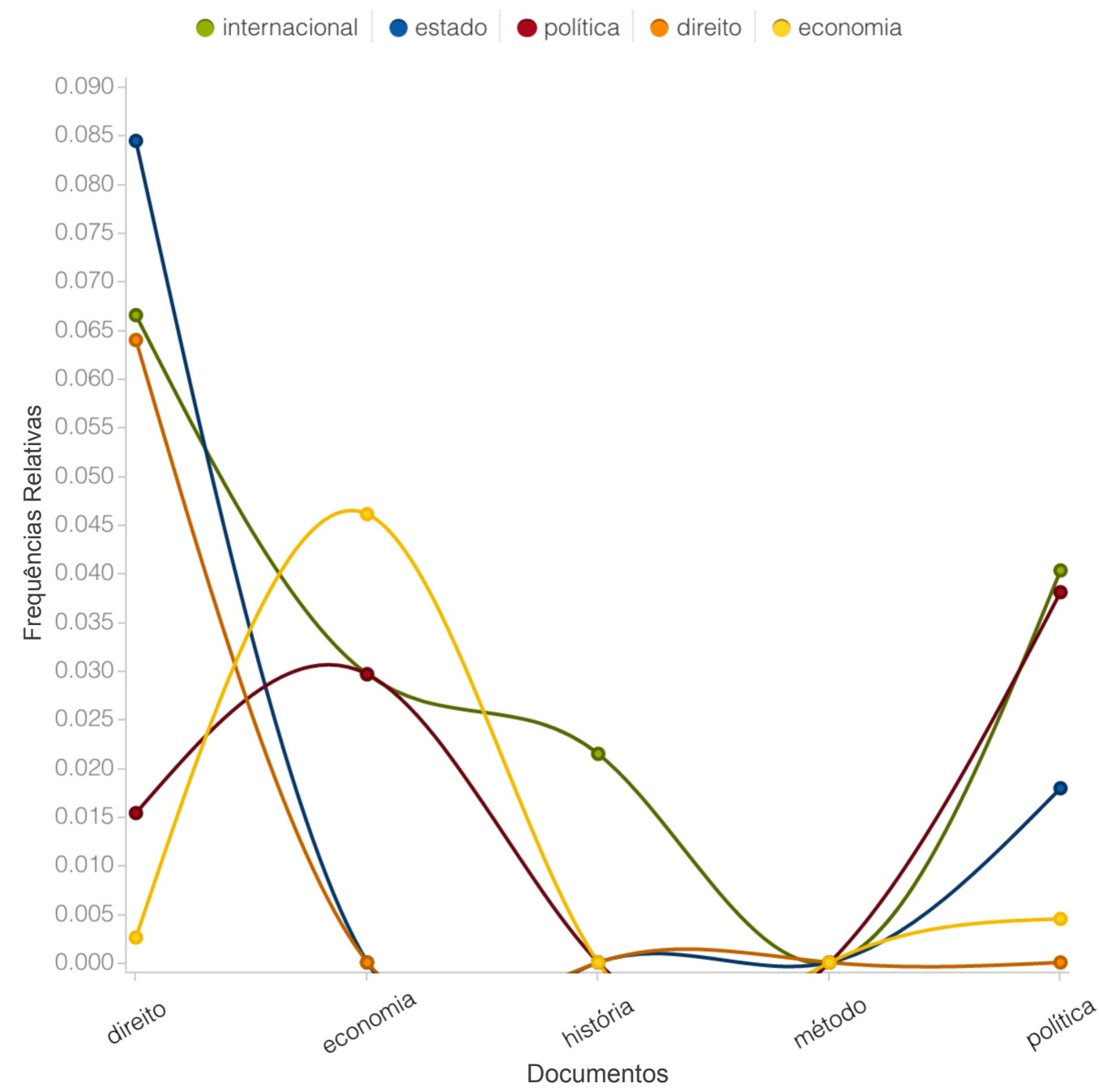

Figura 1 - Frequência relativa das cinco palavras mais usadas por área. 


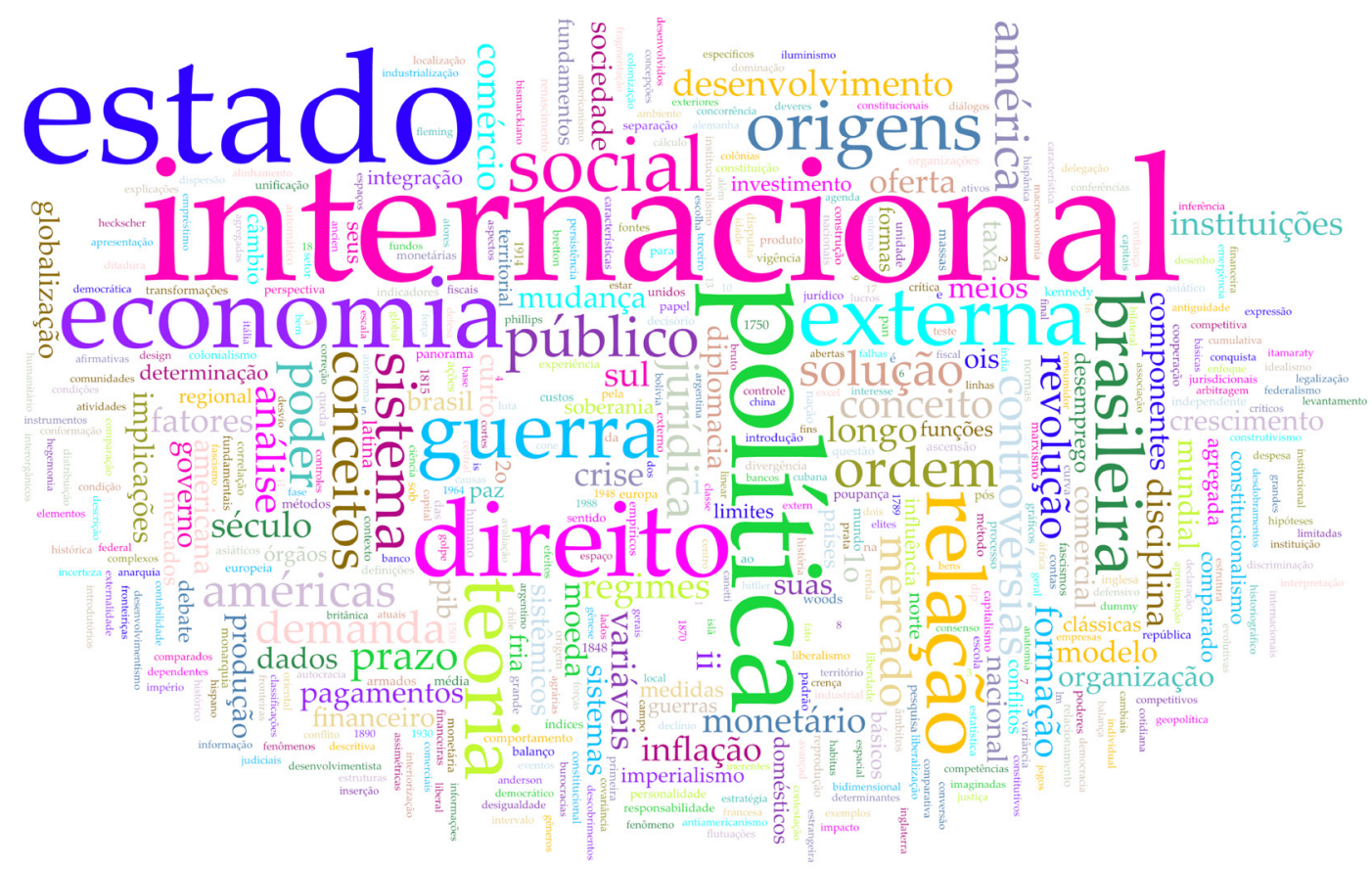

Figura 2 - Quinhentas palavras mais frequentes nas disciplinas obrigatórias em 2016.

A Figura 3 identifica a relação entre as palavras mais frequentes nos programas das disciplinas obrigatórias. No centro, encontra-se a palavra "internacional", com ligação para "política", "economia" e, especialmente, "direito". Além de "internacional", "economia" relaciona-se com "política", que, por sua vez, tem uma relação mais forte com "externa" e "brasileira". "Direito" relaciona-se com "público" e "estado". A área de História ainda não obtém tanta representatividade nas disciplinas obrigatórias. Por outro lado, há temas tratados somente pela Economia, como "financeiro"; Direito com "público", "jurídica" e "estado"; Política com "externa", "comercial" e "brasileira", o que indica a falta de interdisciplinaridade entre as principais áreas, apesar da forte multidisciplinaridade.

O Anexo II discrimina as figuras de dispersão das 55 palavras mais frequentes e os mapas das ligações entre as principais palavras para cada área em 2016. As áreas de Economia e Política apresentam uma distribuição mais heterogênea das principais palavras. Em contrapartida, História e Métodos estão concentradas em algumas poucas palavras-chave. A área de Economia é também a que revela maior diversidade nas conexões das palavras-chave.

\section{Disciplinas Optativas Eletivas}

A Figura 4 exibe a frequência das cinco principais palavras nos conteúdos programáticos das disciplinas optativas eletivas por ano, desde 2006 (ponderada pelo número de alunos que escolheram o curso).

Há a manutenção de um grande interesse por temas relacionados com História, apesar de perder espaço para uma maior diversidade de assuntos nos anos mais recentes. Como as demais palavras não aumentam significativamente sua frequência no período, observa-se um esforço por maior diversidade nas disciplinas optativas oferecidas e em seus conteúdos programáticos.

No Anexo III, apresentamos os mapas anuais das 55 palavras mais frequentes no programa dessas disciplinas. Ao contrário das obrigatórias, notase uma forte presença de "história" nos programas escolhidos pelos alunos. Apesar de "estado" estar presente em todo o período, a palavra "direito" aparece com força somente a partir de 2010 e permanece assim até 2016. Por outro lado, "política" e "economia" estão sempre presentes, tanto nas optativas, quanto nas obrigatórias. Em relação às regiões estudadas pelos alunos, observa-se maior diversidade quando eles têm a possibilidade de escolher as disciplinas. 


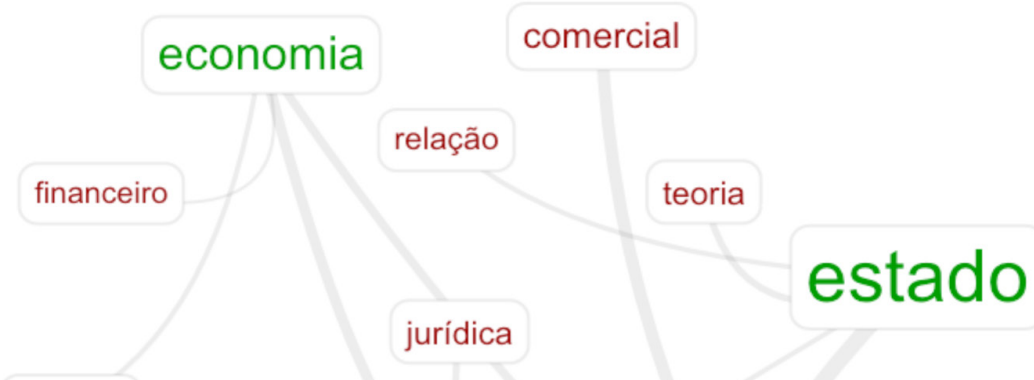

sistema

\section{internacional}

externa

disciplina

contexto

política

brasileira público

\section{direito}

Figura 3 - Ligações entre as palavras mais frequentes das disciplinas obrigatórias.

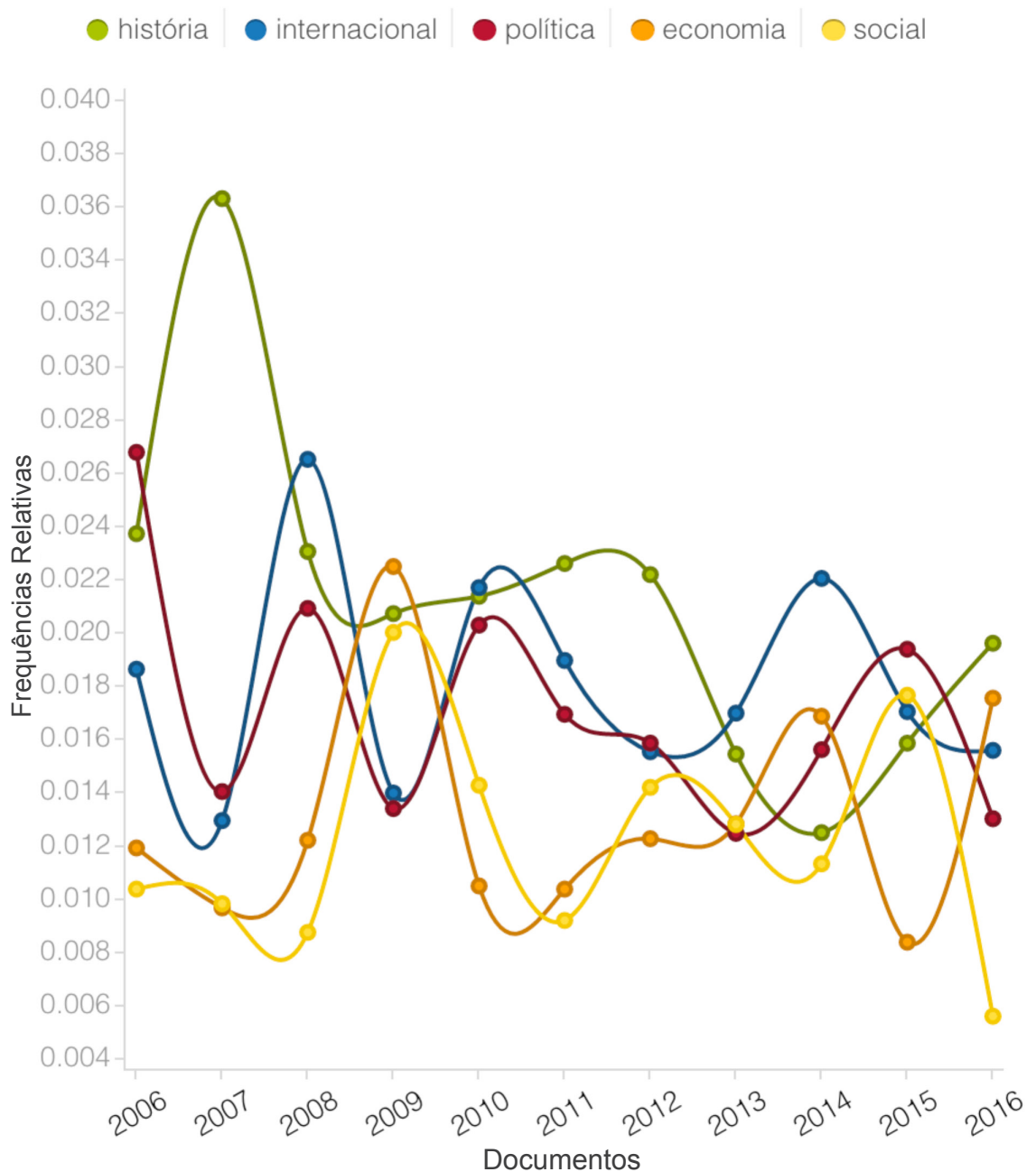

Figura 4 - Cinco palavras mais frequentes nas disciplinas optativas eletivas por ano. 
"Social" tem frequência elevada no conteúdo programático das optativas em todos os anos. "Revolução", "comércio", "desenvolvimento" e "democracia" têm aparecido em vários anos. É interessante relatar o surgimento de temas específicos: "saúde" em 2013 e 2014, "segurança" em 2014, e "ambiente" em 2015, confirmando a diversidade das disciplinas escolhidas de acordo com o contexto internacional.

Por último, os programas das disciplinas optativas eletivas cursadas pelos alunos de 2006 a 2009 foram agregados e contrapostos aos programas das cursadas de 2013 a 2016. Investiga-se se há alguma tendência a temas escolhidos. Considerando a frequência das cinco principais palavras entre os períodos inicial e final estudados, "História", por exemplo, apesar de se manter como o tema bastante recorrente, teve a maior redução, seguido por "Política", ampliando a possibilidade de maior diversidade de temas ou disponíveis ou escolhidos pelos alunos. $\mathrm{O}$ cálculo das quinhentas palavras mais frequentes no conjunto dos conteúdos programáticos das disciplinas cursadas pelos alunos nesses períodos gerou o conjunto de mapas apresentado na Figura 5.

"Direito" está com destaque apenas nos anos finais. Já as demais áreas (História, Política, Economia) aparecem com forte frequência em todo o período. Em relação aos países, as regiões estudadas permanecem as mesmas; e a alguns conceitos, aparecem com destaque nos dois períodos, como "social", "desenvolvimento", "guerra", "comércio", "revolução", "democracia", "crise".

A contraposição de períodos revela que a diversidade de palavras aumentou nos anos mais recentes, pois há menos palavras com tanto destaque, especialmente ao considerar aquelas não relacionadas com as áreas centrais do curso.

\section{Análise das Ligações entre as Palavras mais Frequentes por Ano}

A Figura 6 contrapõe os mapas entre os períodos inicial (2006 a 2009) e final (2013 a 2016) da ligação entre as principais palavras dos conteúdos programáticos das disciplinas cursadas pelos alunos, além da força dessa ligação. Os mapas anuais, resultantes das disciplinas optativas eletivas, estão dispostos no Anexo IV.

Observa-se que o mapa é bastante diferente entre as disciplinas optativas e as obrigatórias, conforme mostra a Figura 3, comentada anteriormente, e a Figura 6. Os dois comportamentos mais relevantes são referentes a "Estado", que está presente nas obrigatórias, mas quase sem força nas optativas, e "História", que, por outro lado, nem aparece nas obrigatórias e é sempre presente nas eletivas.

Em relação às principais ligações, observa-se maior frequência da conexão de "História" com "dialética" e "contemporânea".Já "Política" aparece, na maioria das vezes, conectada com "externa" e "internacional", sugerindo o objeto de estudo. Em 2009, conectou-se com "social" e "Economia", de acordo com o contexto do momento. "Direito" liga-se essencialmente com "internacional", mas a partir de 2013 há ligação com "humano". Por sua vez, "Economia", liga-se mais fortemente com "internacional" e "comércio". A contraposição dos anos agregados do início e final do período revela que "História" diversifica suas ligações nos anos mais recentes, enquanto "Política" perde sua forte conexão com "Internacional" nos últimos anos.

Esses resultados sugerem haver uma ligação relativamente baixa entre as disciplinas que compõem os pilares teóricos do curso (Giência Política, Economia, Direito e História), tendência semelhante à observada no estudo feito com as disciplinas obrigatórias. Em que pese o fato de ter sido utilizada uma metodologia única para fazer esta primeira análise da estrutura curricular, as evidências empíricas apontam para um grau moderado de interdisciplinaridade no curso.

A observação das trajetórias estabelecidas pelos graduandos do Instituto após a realização das disciplinas obrigatórias, como foi visto, é parte imprescindível da análise da composição curricular efetivamente cursada por eles. Um espectro bastante diversificado de disciplinas se abre para as opções. No entanto, é necessário considerar que há fatores do lado da oferta de disciplinas que interferem no processo de 


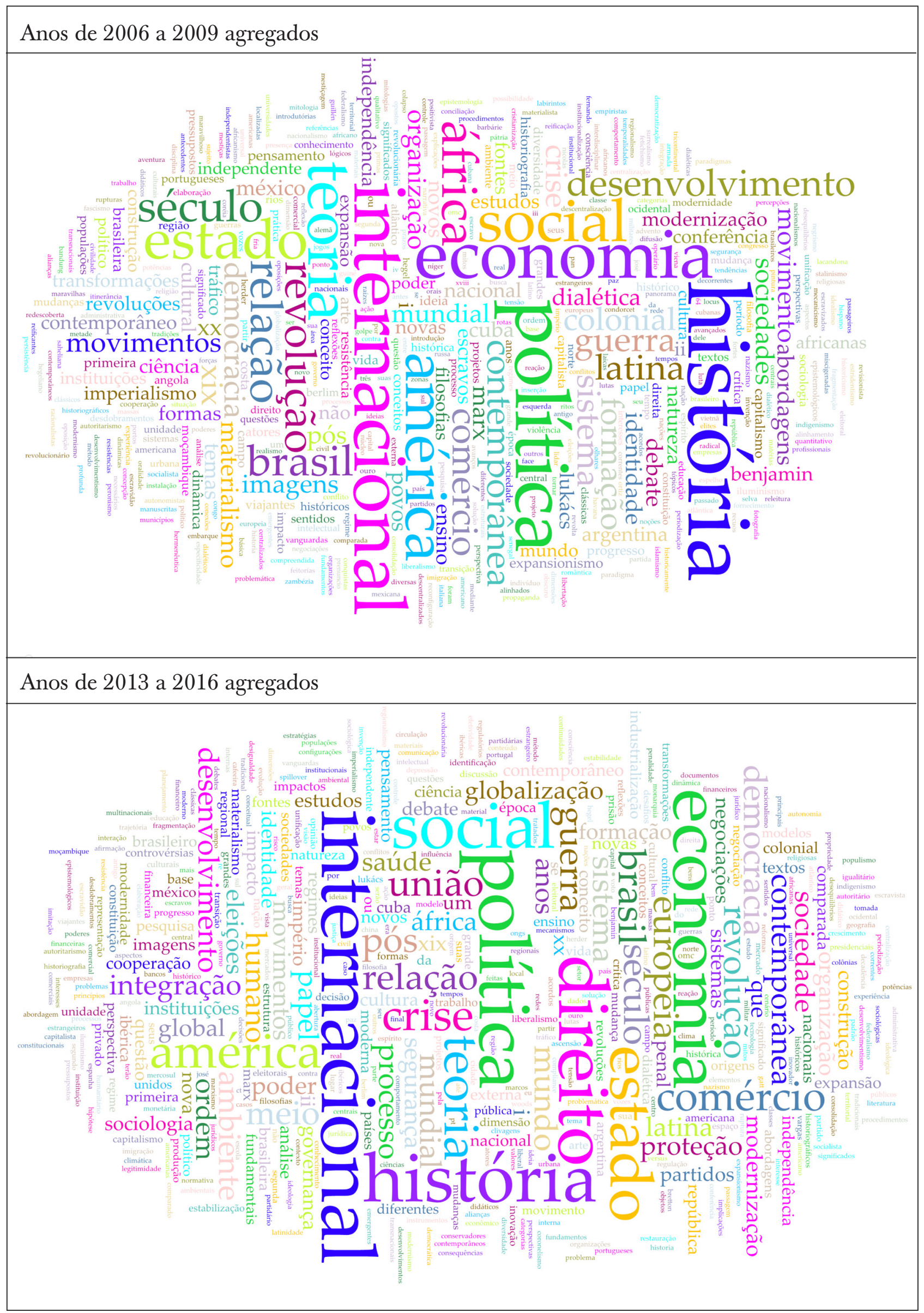

Figura 5 - Quinhentas palavras mais frequentes nas disciplinas optativas eletivas no início e final de períodos. 


\section{Anos de 2006 a 2009 agregados}

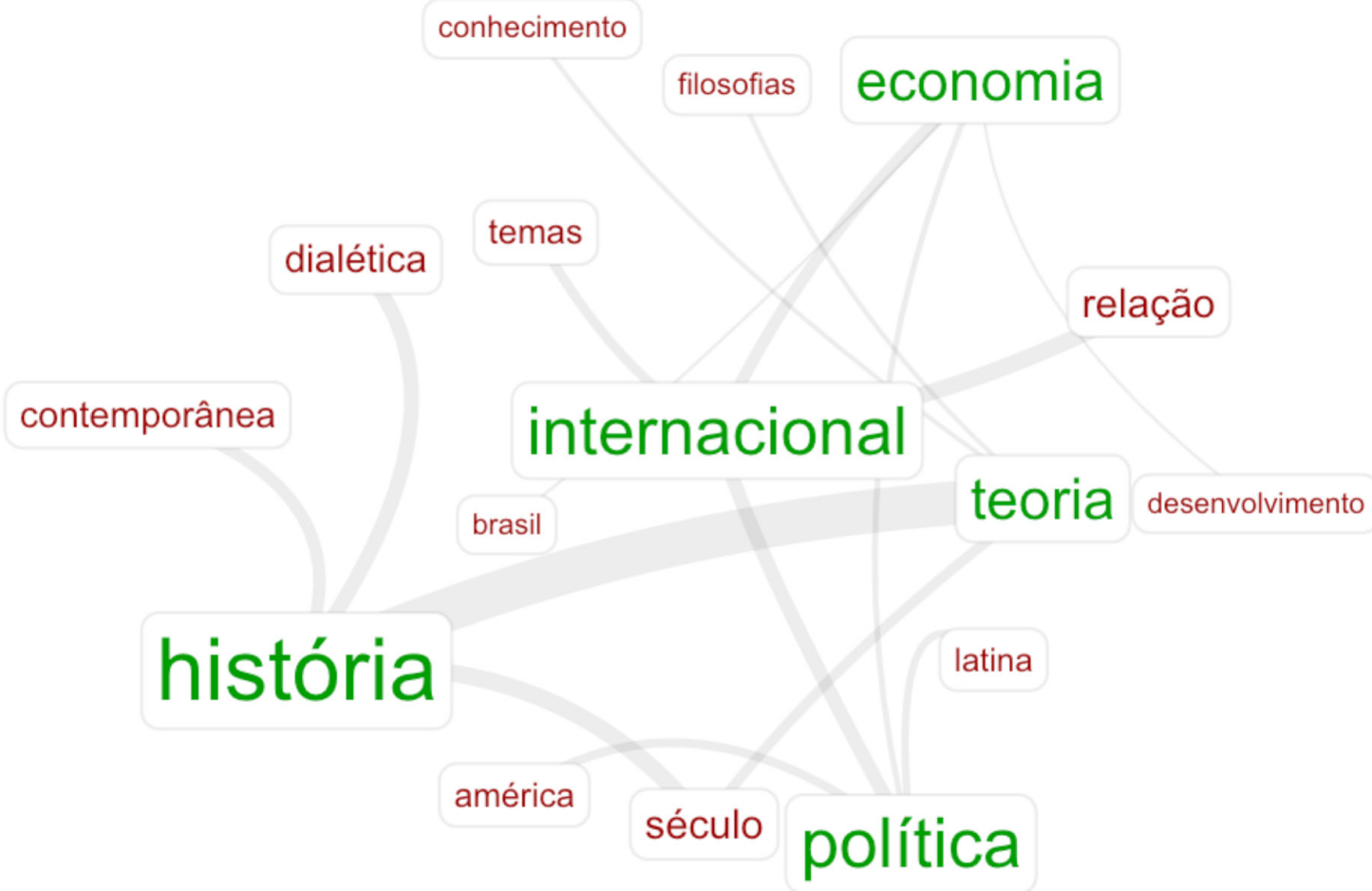

Anos de 2013 a 2016 agregados

\section{política}

latina

meio século

social

\section{internacional}

ambiente história

américa

humano

união

teoria

economia revolução

fundamentais

\section{direito}

contemporânea

Figura 6 - Ligação entre as palavras dos conteúdos programáticos por períodos agregados. 
seleção dos estudantes, como a disponibilidade de vagas para alunos de relações internacionais em outras unidades da USP e também mudanças em seu oferecimento pelos professores.

Mesmo assim, a própria seleção de disciplinas traz uma informação muito relevante para o estudo da adesão dos alunos à proposta curricular de forma ampla. Quando os estudantes têm a liberdade para realizar escolhas, elas tendem a se concentrar nas áreas de formação do curso.

\section{Conclusões e Considerações}

O currículo do BRI-USP foi analisado como um caso situado em uma área de conhecimento cuja origem está na interação entre disciplinas com temas, objetos e metodologias tradicionalmente bem delimitados. Tendo em conta que a estrutura do curso foi concebida a partir da ideia do diálogo entre disciplinas e de manter um equilíbrio na distribuição de disciplinas entre as áreas teóricas de conteúdo, a motivação inicial do trabalho foi investigar, passados quinze anos desde a criação da primeira turma, que tipo de relação se estabeleceu entre as áreas.

Entendemos que a interdisciplinaridade não se reflete apenas na composição de disciplinas individuais e que a estrutura curricular como um todo, a presença das "áreas de base" entre as matérias obrigatórias e a própria convivência dos estudantes com professores de diferentes formações são elementos importantes na formação de indivíduos "diferentes" daqueles que cursam as carreiras tradicionais. No entanto, dadas as dificuldades práticas de se medirem os efeitos das transformações na estrutura pedagógica e no corpo discente, propusemos um exercício para avaliar os programas das disciplinas de graduação, como uma aproximação de seu conteúdo.

A técnica de mineração de textos foi aplicada aos programas das disciplinas obrigatórias e optativas do curso, estudando também as interligações entre seus programas. Os resultados sugerem que, apesar de ter havido ao longo do tempo convergência entre os conteúdos das disciplinas de formação, ainda existe pouca ligação entre elas. No que concerne à demanda dos estudantes por disciplinas optativas, o exercício nos permite responder positivamente a uma das questões que inicialmente nos colocamos sobre o grau de adesão dos alunos às áreas de formação do curso. Ainda que se considere haver um "direcionamento natural" dos estudantes para disciplinas oferecidas por departamentos que respondem por parte substantiva das disciplinas disponíveis e aceitas, o perfil do aluno representativo é marcado por uma tendência a permanecer dentro dos campos disciplinares de formação.

Todavia, a técnica não nos permite fazer um diagnóstico detalhado do grau, tipo e dinâmica da interação entre disciplinas no bacharelado do IRI. Mas se pode afirmar que, ainda que tenha havido uma intensificação da relação entre as disciplinas, as evidências apontam para um grau moderado de interdisciplinaridade no curso.

\section{Notas}

1 a, as, o, os, e, em, na, nas, no, nos, para, da, das, do, dos, de, novo, nova, novos, novas, sobre, entre, com, uma, como, i), ii), iii), ", ', -, ., , , ?.

2 Software gratuitamente disponível na internet: http://voyant-tools.org/.

\section{Referências Bibliográficas}

AALTO, Pami. "Organizing Interdisciplinary International Studies: From Puzzlement to Research Programmes". In: AALTO, Pami; HARLE, Vilho \& MOISIO, Sami (eds.). International Studies. Interdisciplinary Approaches. New York: Palgrave Macmillan, 2011.

BLANTON, Robert G. "Surveying International Studies Programs: Where Do We Stand?". International Studies Perspectives, vol. 10, 2009, pp. 224-240.

JACOBS, Jerry A. In Defense of Disciplines: Interdisciplinarity and Specialization in the Research University. Chicago: The University of Chicago Press, 2014.

KLEIN, Julie Thompson. Humanities, Culture, and Interdisciplinarity: the Changing American Academy. New York: State University of New York Press, 2005.

Creating Interdisciplinary Campus Cultures: a Model for Strength and Sustainability. San Francisco: Jossey Bass, 2010a.

"A Taxonomy of Interdisciplinarity". In: FRODEMAN, Robert; KLEIN, Julie Thompson \& PACHECO, Roberto Carlos dos Santos. The Oxford Handbook of Interdisciplinarity. Oxford: Oxford University Press, 2010b. 
LATTUCA, Lisa R. Creating Interdisciplinarity: Interdisciplinary Research and Teaching among College and University Faculty. Nashville: Vanderbilt University Press, 2001.

LONG, David. "Interdisciplinarity and the Study of International Relations". In: AALTO, Pami; HARLE, Vilho \& MOISIO, Sami (eds.). International Studies. Interdisciplinary Approaches. New York: Palgrave Macmillan, 2011.

RIGUEIRA, Paulo. "Relações Internacionais como Disciplina". Relações Internacionais, Lisboa, n. 36, dez. 2012, pp. 23-46.

SMITH, Steve. "The Discipline of International Relations: still an American Social Science?". British Journal of Politics and International Relations, vol. 2, n. 3, out. 2000, pp. 374-402.
VELHO, G. "O Lugar da Interdisciplinaridade". In: FUNDAÇÃO GETÚlIO VARGAS. Centro de Pesquisa e Documentação de História Contemporânea do Brasil. CPDOC 30 anos. Rio de Janeiro: Ed. Fundação Getulio Vargas-CPDOC. 2003.

VENTURA, Deisy de F. L. \& LINS, Maria Antonieta D. T. "Educação Superior e Complexidade: Integração entre Disciplinas no Campo das Relações Internacionais". Cadernos de Pesquisa, vol.151, 2014, pp. 104-131.

WEINGART, Peter. "A Short History of Knowledge Formations". In: FRODEMAN, Robert KLEIN, Julie Thompson \& PACHECO, Roberto Carlos dos Santos. The Oxford Handbook of Interdisciplinarity. Oxford: Oxford University Press, 2010.

Publicado em 12/06/2018.

\section{Anexos}

\begin{tabular}{|c|c|}
\hline Lista das palavras agregadas... & ... em um único vocábulo \\
\hline contemporânea, contemporâneas, contemporâneos & contemporâneo \\
\hline Sistemas & sistema \\
\hline brasileiro, brasileiros, brasileiras & brasileira \\
\hline estados & estado \\
\hline sociais & social \\
\hline democracias & democracia \\
\hline teorias & teoria \\
\hline $\begin{array}{l}\text { econômicos, econômico, econômicas, econômica, } \\
\text { macroeconomia, microeconomia, macroeconômica, } \\
\text { microeconômica, macroeconomias, microeconomias, } \\
\text { macroeconômicas, microeconômicas, macroeconômico, } \\
\text { microeconômico, macroeconômicos, microeconômicos }\end{array}$ & economia \\
\hline humanos, humanas & humano \\
\hline internacionais & internacional \\
\hline políticas, políticos, político & política \\
\hline relações & relação \\
\hline direitos & direito \\
\hline crises & crise \\
\hline
\end{tabular}

Anexo I - Palavras agregadas a um único vocábulo. 


\begin{tabular}{|c|c|}
\hline Direito & Economia \\
\hline 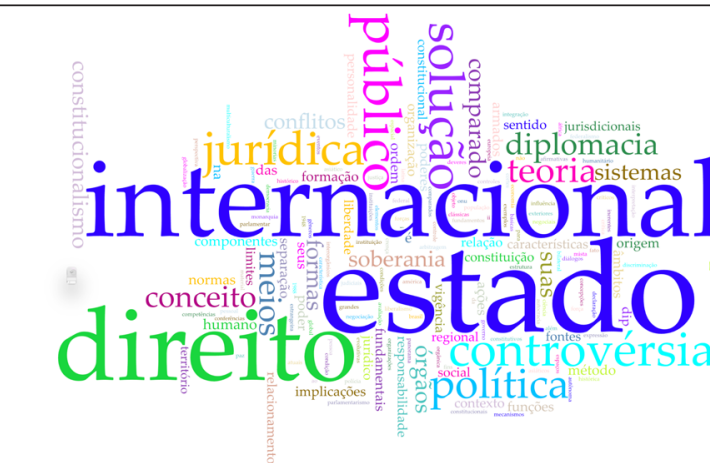 & 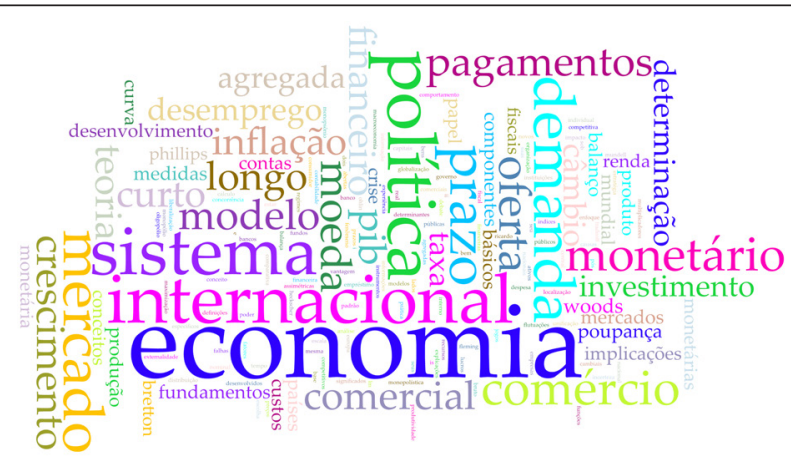 \\
\hline História & Método \\
\hline 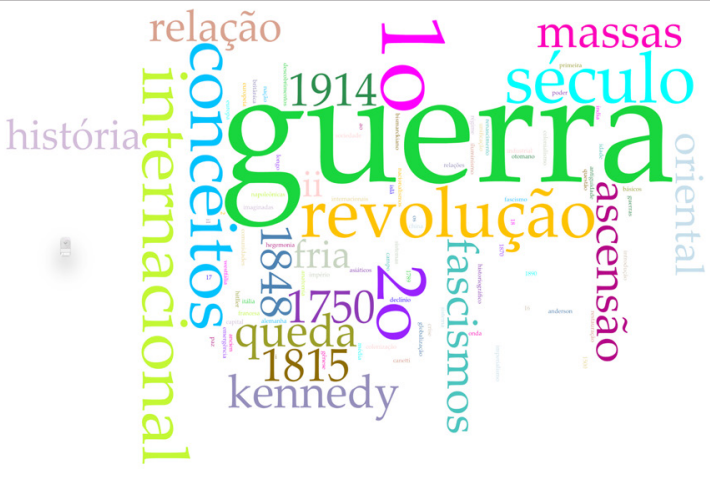 & 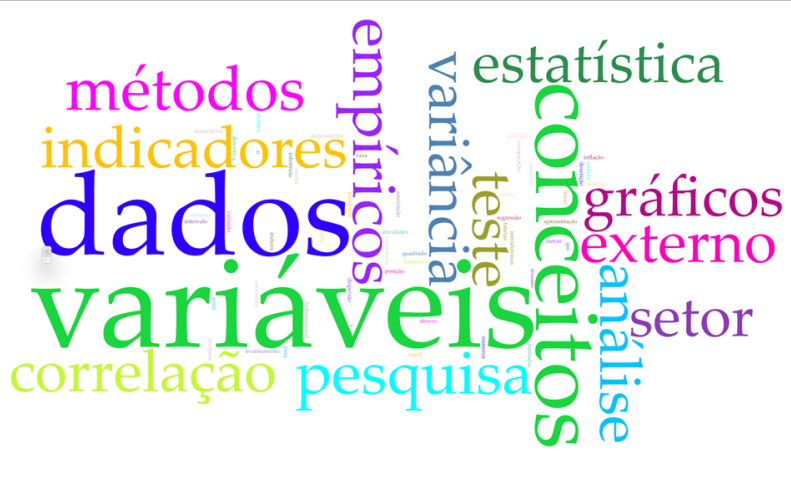 \\
\hline \multicolumn{2}{|l|}{ Política } \\
\hline 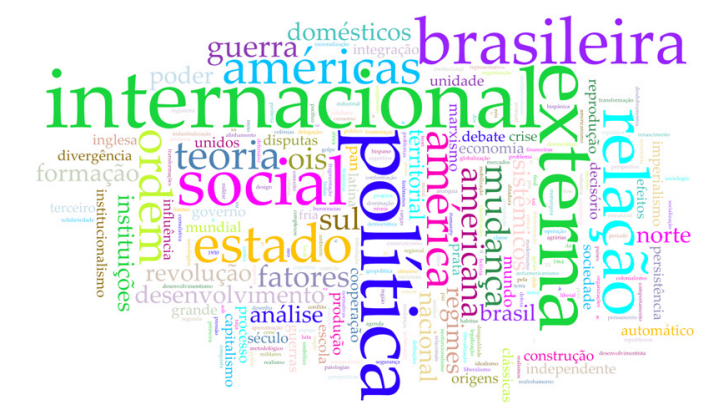 & $\begin{array}{c}\text { Espaço intencionalmente } \\
\text { deixado em branco pela } \\
\text { diagramação }\end{array}$ \\
\hline
\end{tabular}

Mapa das Ligações

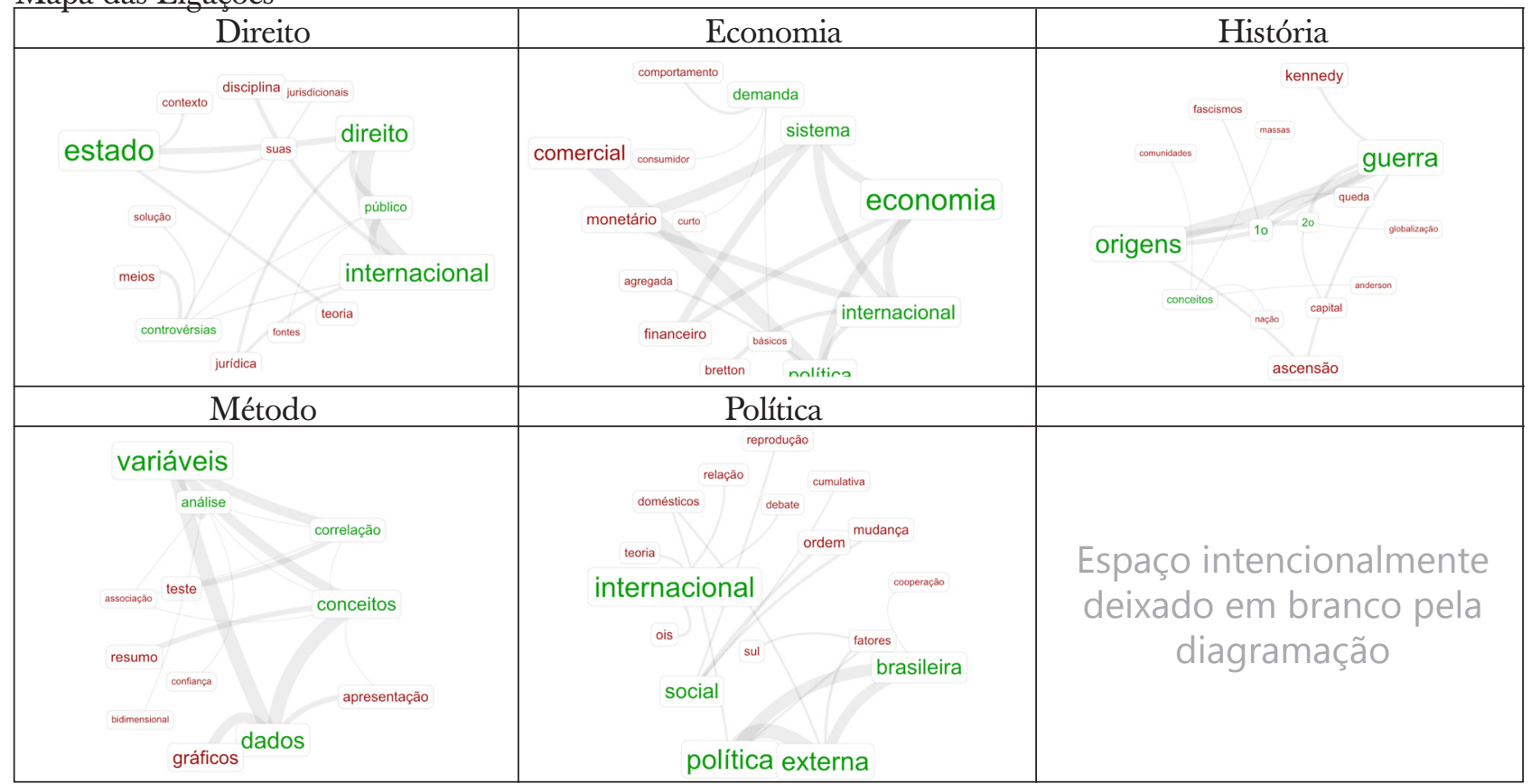

Anexo II - 55 palavras mais frequentes e mapa das ligações - por área das disciplinas obrigatórias, 2016. 


\begin{tabular}{|c|c|c|}
\hline 2006 & 2007 & 2008 \\
\hline 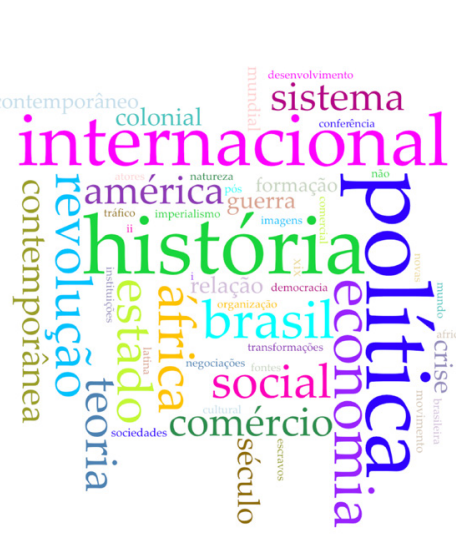 & 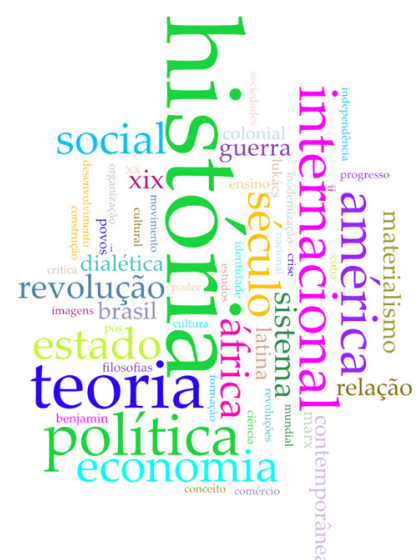 & 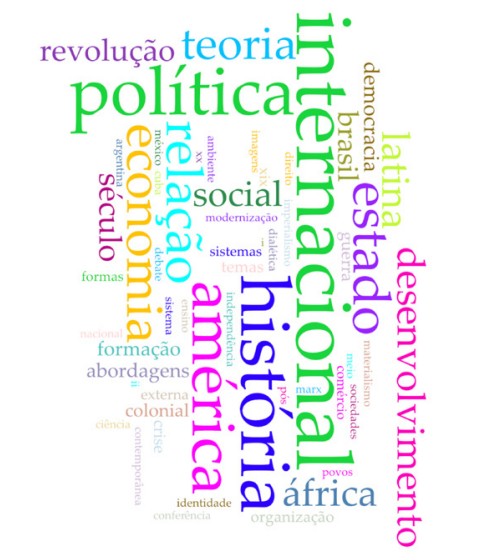 \\
\hline 2009 & 2010 & 2011 \\
\hline 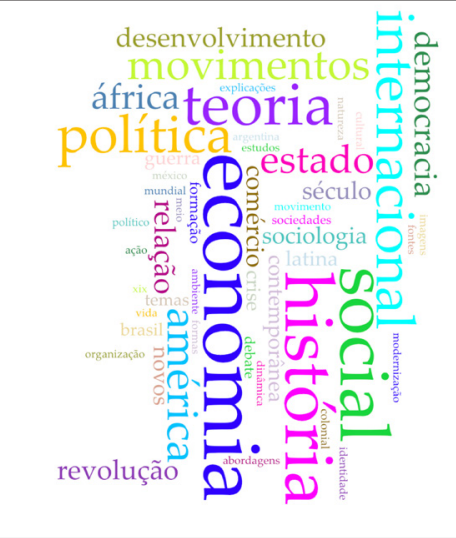 & 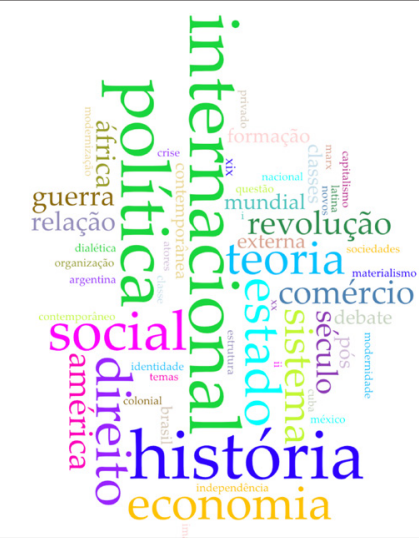 & 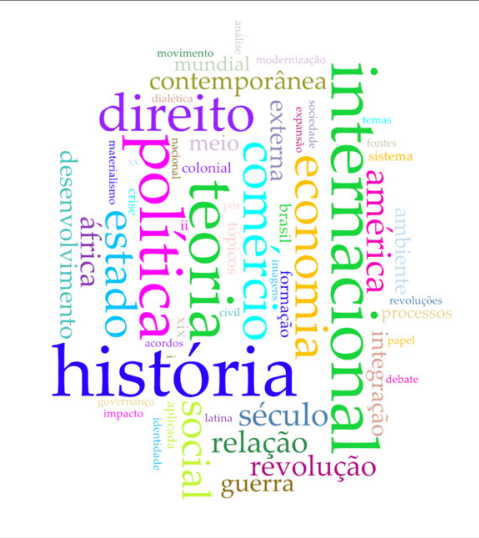 \\
\hline 2012 & 2013 & 2014 \\
\hline 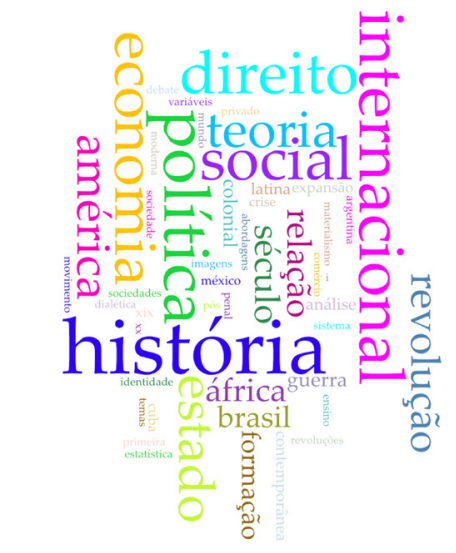 & 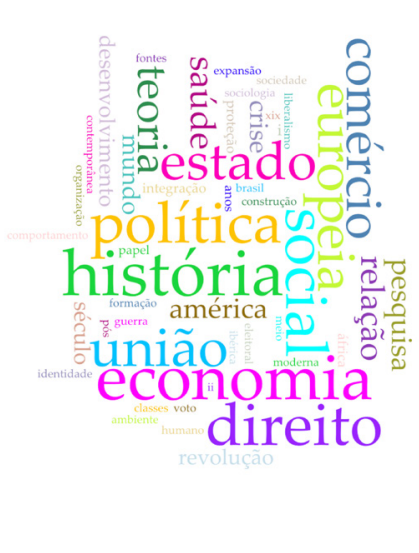 & 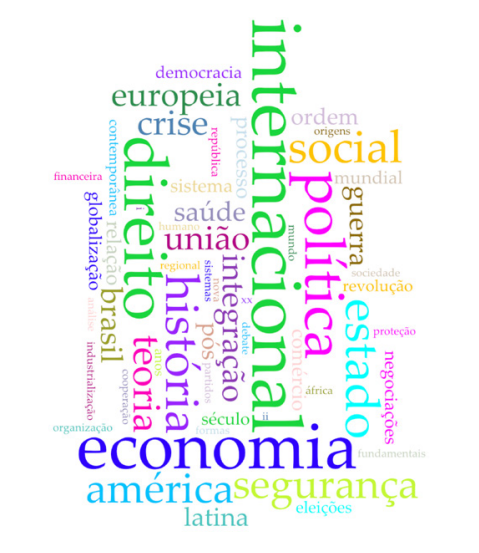 \\
\hline 2015 & 2016 & \\
\hline 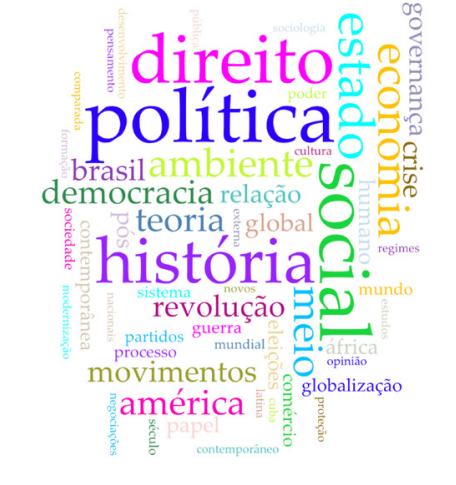 & 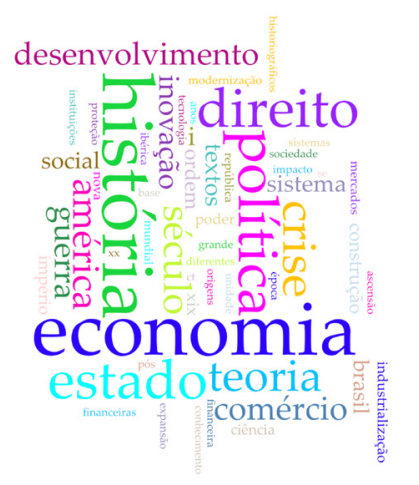 & $\begin{array}{c}\text { Espaço intencionalmente } \\
\text { deixado em branco pela } \\
\text { diagramação }\end{array}$ \\
\hline
\end{tabular}

Anexo III - 55 palavras com maior frequência no conteúdo programático das disciplinas optativas eletivas por ano. 


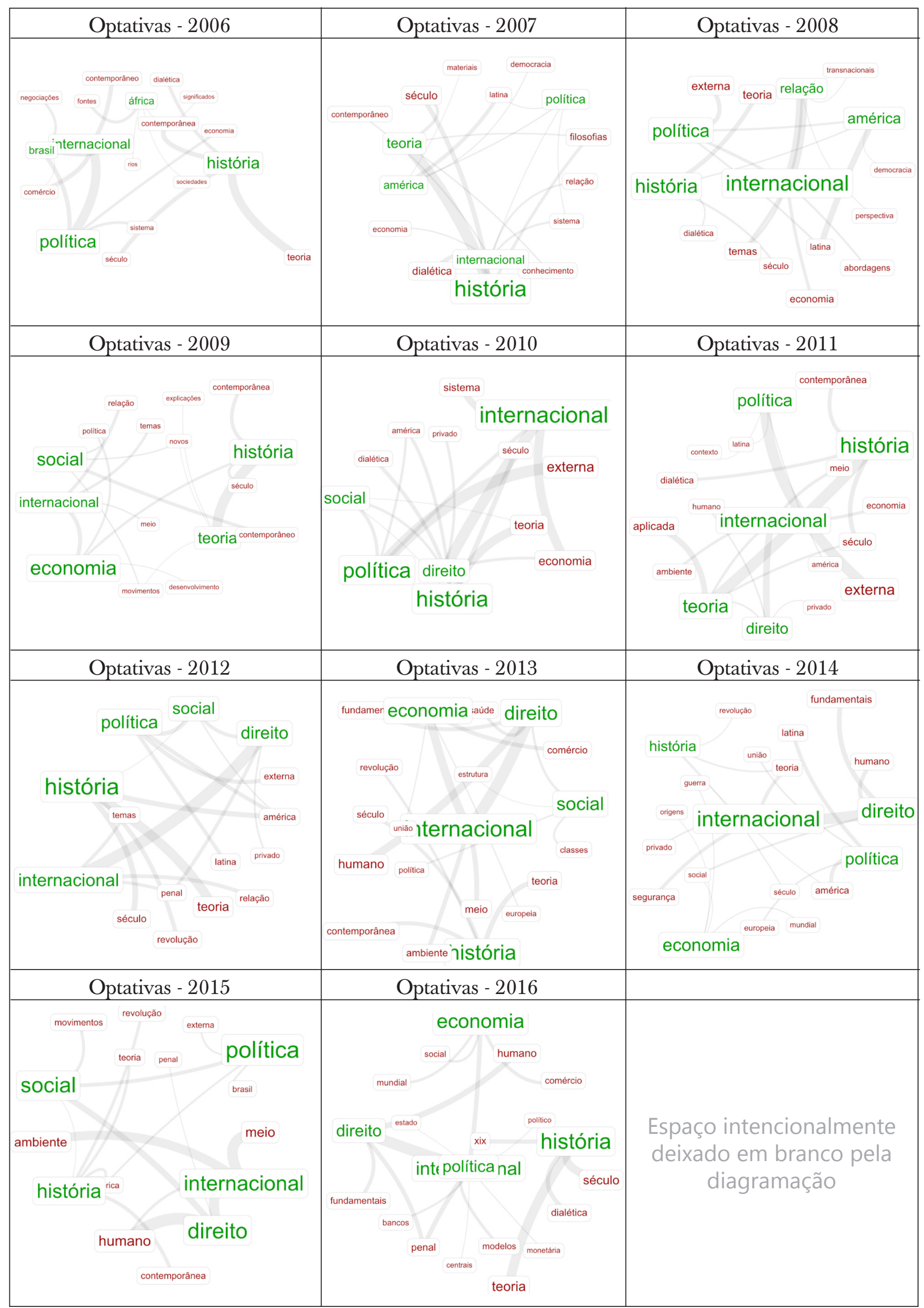

Anexo IV - Ligação entre as 55 palavras com maior frequência no conteúdo programático das disciplinas optativas eletivas por ano. 